\title{
LXXV. The motion of electrons in solids. Part I.-Electric conductivity, Kirchhoff's law and radiation of great wave-length
}

\section{J.H. Jeans M.A. F.R.S.}

To cite this article: J.H. Jeans M.A. F.R.S. (1909) LXXV. The motion of electrons in solids. Part I.-Electric conductivity, Kirchhoff's law and radiation of great wave-length, Philosophical Magazine Series 6, 17:102, 773-794, DOI: 10.1080/14786440608636657

To link to this article: http://dx.doi.org/10.1080/14786440608636657

曲 Published online: 21 Apr 2009.

Submit your article to this journal $[\pi$

Џll Article views: 1

Q View related articles $₫$

47 Citing articles: 5 View citing articles 
LONDON, EDINBURGH, AND DUBLIN

\title{
PHILOSOPHICAL MAGAZINE
}

\author{
AND \\ JOURNAL OF SCIENCE.
}

[SIXTH SERIES.]

$J U N E 1909$.

LXXV. The Motion of Electrons in Solids. Part I.-Electric Conductivity, Kirchhoff's Law and Radiation of Great Wave-length. $\quad B y$ J. H. JeANs, M.A., F.R.S., Professor of Applied Mathematics in Princeton University *.

1. THE Electron-theory of metals bas been developed 1 by Drude, Thomson, Lorentz, and others. The work of all these writers postulates a motion consisting of free paths and collisions. These and other conceptions imported from the Kinetic Theory of Gases have led very successfully to a general interpretation of many of the phenomena of solids, but cannot be expected to lead to accurate quantitative results. I have therefore thought it would be of value to try to develop a theory which shall be free from all suppositions for which there is not direct numerical justification. Such a theory, if it can be constructed, ought to lead not only to predictions of the nature of the phenomena with which it is concerned, but also to an exact evaluation of the quantities involved in these phenomena.

\section{Electric Conductivity.}

2. In a conductor which is under the influence of no externally-impressed electric force, we suppose that there are $\mathrm{N}$ electrons per unit volume, moving with the velocities

$$
\text { * Communicated by the Author. }
$$

Phil. Mag. S. 6. Vol. 17. No. 102. June 1909. 
assigned to them by Maxwell's law ${ }^{*}$. At a temperature T given by $\mathrm{RT}=\frac{1}{2 h}$, the law of distribution of velocities is

$$
\mathrm{N} \sqrt{\frac{h^{3} m^{3}}{\pi^{3}}} e^{-h m\left(u^{2}+v^{2}+w^{2}\right)} d u d v d w . . .
$$

It an electric force $\mathrm{X}$ is brought into play parallel to $\mathrm{O} x$, this distribution will immediately be altered. Each electron will acquire momentum parallel to $\mathrm{O} x$ at a rate $\mathrm{X} e$, but this gain in momentum will be held in check by a perpetual transfer of momentum between each electron and all the molecules by which it is influenced at any instant.

Under law (1), the average value of $u$ is zero. Under the new law, the average of $u$ will have some value $u_{0}$, different from zero. Corresponding to any value of $u_{0}$, there is a current $i_{x}$ parallel to $\mathrm{O} x$ of amount

$$
i_{x}={\mathrm{N} e u_{0}} \text {. . . . . . . . }
$$

3. A brief calculation will show that for all values of $\mathrm{X}$ with which we shall be concerned, $u_{0}$ is small in comparison with the average numerical values of $u$. We may perfectly legitimately neglect squares of $u_{0}$, and, in particular, in calculating any quantity which has ultimately to be multiplied by $u_{0}$, we may assume formula (1) to give the distribution of velocities.

4. We proceed now to calculate an expression for the transfer of momentum between electrons and molecules. We shall do this first, for simplicity, upon the supposition that the motion consists of free paths and collisions. Afterwards we shall find a perfectly general expression.

Consider an electron approaching a molecule with a velocity of components $u, v, w$. It will describe a curved orbit having its free path before collision and its free path after collision as asymptotes. Its loss of momentum at collision will depend on $u, v, w$, on the orientation of the molecule, and on the position in which the free path before collision meets a perpendicular plane through the centre of the molecule.

We know that all positions are equally likely for this latter point, and if the solid is isotropic all orientations are equally likely for the molecule. On averaging, we can find the probable loss of momentum at collision. Clearly this average

* It is important to remember that Maxwell's law gives the partition of velocities for particles in collision or acted on by any field of force, as well as when on a free-path. The existence of a field of force alters the distribution of density, but not of velocities ( $c f$. the author's 'Dynamical Theory of Gases,' p. 78). 
loss of momentum, regarded as a vector, can have no direction except that of the initial velocity $u, v, w$ : its amount will depend on the constants of the electron and molecules and on $u^{2}+v^{2}+w^{2}$, but not on $u, v$, and $w$ separately. We can accordingly suppose that the original velocity $u, v, w$ is reduced by collision to

$$
(1-\alpha) u, \quad(1-\alpha) v, \quad(1-\alpha) w,
$$

where $\alpha$ depends on $u^{2}+v^{2}+w^{2}$ and constants only.

In a time $d t$ which is large compared with the time of a collision, the loss to the momentum of the $\mathrm{N}$ electrons is therefore of the form

$$
\mathrm{N}_{\gamma} u_{0} d t, \quad \mathrm{~N} \gamma v_{0} d t, \quad \mathrm{~N}_{\gamma} w_{0} d t,
$$

where $u_{0}, v_{0}, w_{0}$ are average values of $u, v, w$ and $\gamma$ depends on $u^{2}+v^{2}+w^{2}$ and constants only.

We accordingly have the equation

$$
\frac{d}{d t}\left(\mathrm{~N} m u_{0}\right)=\mathrm{NX} e-\mathrm{N} \gamma u_{0}, \quad . \quad . \quad .
$$

provided the interval of time $d t$ is taken to be large compared with the time of collision.

5. Let us now carry out the corresponding calculation without assuming the existence of free paths. We fix our attention on all the electrons of which the velocity-components at a given instant $t=0$ lie within a small range $d u d v d w$ surrounding the values $u, v, w$. If there were no externally applied electric force, the law of distribution of these electrons in space would be

$$
\mathrm{NA} e^{-2 h x} d x d y d z \quad \cdot \quad \cdot \quad \cdot
$$

where $\chi$ is the potential energy of an electron at the point $x, y, z$ and $\mathrm{A}$ is such that

$$
\text { A } \iiint e^{-2 h x} d x d y d z=1,
$$

the integral being taken throughout a unit volume. With an electric force $\mathrm{X}$ acting, the law will be different from this by terms of the order of $\frac{u_{0}}{u}$, but these terms may be neglected whenever their retention would lead to ultimate terms of the order of $\frac{u_{0}^{2}}{u^{2}}$.

Assuming (4) to be the law of distribution of these electrons in space, we calculate the values of $u, v, w$ after time $\tau$, corresponding to all initial positions of the electrons, $3 \mathrm{G} 2$ 
and average according to the law (4). In this way we find the average velocity components $u^{\prime}, v^{\prime}, w^{\prime}$ after time $\tau$. The vector $u^{\prime}, v^{\prime}, w^{\prime}$ must, from symmetry, be in the same direction as $u, v, w$ : its amount will depend on $u^{2}+v^{2}+w^{2}$ and on the constants of the matter and the electrons.

Thus we must have

$$
u^{\prime}=u f\left(\tau, u^{2}+v^{2}+w^{2}\right), \& c . ;
$$

and hence, on averaging for all values of $u, v, w$, we find that if $u_{0}{ }^{\prime}, v_{0}{ }^{\prime}, w_{0}{ }^{\prime}$ are the values of $u_{0}, v_{0}, w_{0}$ after time $\tau$,

$$
u_{0}^{\prime}=u_{0} \phi(\tau, h) \text {. . . . . . }
$$

If we could evaluate $\phi$, this equation would be the generalization, in integral form, of equation (3) with $\mathrm{X}=0$.

We can obtain some information as to the function $\phi$. We notice first that at time $t=0$,

$$
m \frac{d u}{d t}=-\frac{d \chi}{d x}
$$

so that, on averaging, the value of $\frac{d u_{0}}{d t}$ is zero, and hence $\phi(\tau, h)$, for small values of $\tau$, is of the form $1+\tau^{2} f(h)$.

Next we consider the form of the function for large values of $\tau$. Let $\tau$ be so large compared with the time of encounter with a molecule, that the velocities of those electrons which originally had velocities $u, v, w$ may be regarded as distributed at random. Then, if $u_{0}{ }^{\prime \prime}, v_{0}{ }^{\prime \prime}, w_{0}{ }^{\prime \prime}$ are the values of $u_{0}, v_{0}, w_{0}$ after a time $2 \tau$, we shall have, in addition to equation (5),

$$
u_{0}^{\prime \prime}=u_{0} \phi(2 \tau, h) ; \quad u_{0}^{\prime \prime}=u_{0}^{\prime} \phi(\tau, h), \& \mathrm{c} \text {. }
$$

whence it follows that

$$
\phi(\tau)=\mathrm{A} e^{-\mathrm{B} \tau},
$$

and equation (6) can be put in the form

$$
\frac{d u_{0}}{d t}=-\mathrm{B} u_{0}
$$

provided $d t$ is sufficiently large. This equation is exactly of the form of (3) with $X=0$. It follows that the general equation, no matter what the nature of the motion of the electrons, is of the type of equation (3).

6. We now return to equation (3), which with a suitable value for $\gamma$ will express the relation between $\mathrm{X}$ and $u_{0}$ in any motion in which the quantities do not change too rapidly with the time. 
In the steady state in which a steady current $i_{x}$ is maintained by an electric force $\mathrm{X}$, the left-hand member must vinish, so that we have

or by equation (2),

$$
u_{0}=\frac{\mathrm{X} e}{\gamma}
$$

$$
i_{x}=\frac{\mathrm{N} e^{2}}{\gamma} \mathrm{X} . . . . . . .
$$

giving Ohm's law. The conductivity for steady currents, which we shall call $\kappa$, is given by

$$
\kappa=\frac{\mathrm{N} e^{2}}{\gamma} \cdot \text {. . . . . . }
$$

If we replace $u_{0}$ and $\gamma$ in equation (3) by their values as given by equations (2) and (7), we obtain

$$
\frac{d i_{x}}{d t}=\frac{\mathrm{N} e^{2}}{m}\left(\mathrm{X}-\frac{i_{x}}{\kappa}\right) . \quad \text {. . . . . . }
$$

7. Corresponding to a periodic impressed force

$$
\mathrm{X}=\mathrm{X}_{0} \cos p t
$$

the solution of this equation is

where

$$
i_{x}=\kappa \mathrm{X}_{0} \cos (p t-\epsilon) \cos \epsilon, \quad \text {. . . }
$$

$$
\tan \epsilon=\kappa p \frac{m}{\mathrm{~N} e^{2}} .
$$

We can readily calculate the rate at which energy is dissipated by the resistance. In time $d t$ and in volume $d x d y d z$, an amount of electricity $i_{x} d t d y d z$ falls through a potential-difference $\mathrm{X}_{0} \cos p t d x$, its gain in momentum being nil. The work dissipated is accordingly

$$
i_{x} \mathrm{X}_{0} \cos p t d x d y d z d t
$$

so that the loss of energy per unit volume per unit time is

$$
\begin{aligned}
i_{x} \mathrm{X}_{0} \cos p t & =\kappa \mathrm{X}_{0}^{2} \cos \epsilon \cos (p t-\epsilon) \cos p t \\
& =\kappa \mathrm{X}_{0}^{2}\left(\cos ^{2} \epsilon \cos ^{2} p t+\cos \epsilon \sin \epsilon \cos p t \sin p t\right) .
\end{aligned}
$$

The average value of this, averaged over a number of complete periods, is

$$
\frac{1}{2} \kappa \mathrm{X}_{0}^{2} \cos ^{2} \epsilon=\frac{1}{2} \mathrm{X}_{0}^{2} \frac{\kappa}{1+\frac{\kappa^{2} p^{2} m^{2}}{\mathrm{~N}^{2} e^{4}}}
$$


If $c$ is the conductivity for currents of frequency $p$, this expression must be equal to $\frac{1}{2} c \mathrm{X}_{0}^{2}$, and hence we must have

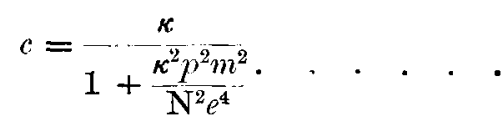

8. For the same quantity $c$, Sir J. J.Thomson, by a different method, obtains

$$
c=\kappa\left(\frac{\sin \frac{1}{2} p t}{\frac{1}{2} p t}\right)^{2}, \quad \text {. . . . . . }
$$

where $t$ is the time of description of a free-path, and is connected with $\kappa$ by the relation

$$
\kappa=\frac{1}{2} \frac{\mathrm{N} e^{2}}{m} t \text {. }
$$

If we eliminate $t$ between this and (11) we get as Thomson's value for $c$,

$$
c=\kappa\left(\frac{\sin \frac{\kappa p m}{\mathrm{~N} e^{2}}}{\frac{\kappa p m}{\mathrm{~N} e^{2}}}\right)^{2}=\kappa\left(1-\frac{1}{3} \frac{\kappa^{2} p^{2} m^{2}}{\mathrm{~N}^{2} e^{4}}+\ldots\right) .
$$

The divergence between this and formula (10) can, I think, be traced to the fact that 'Thomson's system is, so to speak, kinematical and not dynamical. In a dynamical system the time of a free-path must depend on the velocity with which the path is described.

\section{Propagation of Light.}

9. Let $\mathrm{X}$ be the intensity parallel to $\mathrm{O} x$, measured in electromagietic units, and $i_{x}$ the corresponding component of convection-current, connected with $\mathrm{X}$ by equation (8). The total current parallel to $\mathrm{O} x$ is

$$
\begin{aligned}
& \frac{\mathrm{K}}{4 \pi \mathrm{C}} \frac{d \mathrm{X}}{d t}+i_{x}, \\
& \frac{1}{4 \pi}\left(\frac{\partial \gamma}{\partial y}-\frac{\partial \beta}{\partial z}\right) .
\end{aligned}
$$

For waves of frequency $p$, we can take both $\mathrm{X}$ and $i_{x}$ proportional to $e^{i p t}$, so that from equation $(8)$,

$$
i_{x}=\frac{1}{i p\left(\frac{1}{\kappa}+\frac{m}{\mathrm{~N} e^{2}} i p\right)} \frac{d \mathrm{X}}{d t}, . . . .
$$


and the total current (12) becomes

$$
\begin{aligned}
& \frac{\mathrm{K}^{\prime}}{4 \pi \mathrm{C}} \frac{d \mathrm{X}}{d t}, \\
& \mathrm{~K}^{\prime}=\mathrm{K}+\frac{4 \pi \mathrm{C}}{i p\left(\frac{1}{\kappa}+\frac{m}{\mathrm{~N} e^{2}} i p\right)} \quad \cdot \quad \cdot \quad \cdot
\end{aligned}
$$

where

The analysis for the propagation of light is now identical in form with that for the propagation of light in a non-conducting medium of inductive capacity $\mathrm{K}^{\prime}$.

10. It will be seen that the equations arrived at in this way are exactly identical with those given by Drude, although reached in a different manner. Our original differential equations (3) and (8) we found to be true only when the infinitesimal interval $d t$ could be supposed at least as great as the time of a collision. Thus our equations will be true only for light of period much greater than the time of a collision.

\section{Number of Free Electrons per Unit Volume.}

11. As Thomson has noticed ${ }^{*}$, the experiments of Hagen and Rubenst, combined with a formula expressing the variation of conductivity with frequency, will give us information as to the value of $\mathrm{N}$. The velocity of an electron is about $10^{7}$, the radius of an atom about $10^{-8} \mathrm{~cm}$. The time of collision is therefore probably about $10^{-15}$ sec.; for light of wave-length $\lambda=4 \mu$, the time of vibration would be about thirteen times the time of collision. It would therefore appear to be legitimate to use our formula for light of wave-length $4 \mu$ and greater, but probably not for wave-length much less than this.

Rubens and Hagen denote by $\mathrm{C}_{\lambda}$ the product of $(100-\mathrm{R})$ (where $R$ is the reflecting power for wave-length $\lambda$ ) and the square root of $\kappa$, the conductivity for infinite wave-length. They denote by $C_{\lambda}^{\prime}$ the quantity $\frac{36 \cdot 5}{\sqrt{\bar{\lambda}}}$. According to a theory which neglects the variation of conductivity with frequency, $\mathrm{C}_{\lambda}^{\prime}$ ought to be equal to $\mathrm{C}_{\lambda}$. According to our equations $\mathrm{C}^{\prime}$ ought to be equal to $(100-\mathrm{R}) \sqrt{c}$, so that we ought to have

$$
\left(\frac{\mathrm{C}_{\lambda}}{\mathrm{C}_{\lambda^{\prime}}^{\prime}}\right)^{2}=\frac{\kappa}{c}=1+\frac{\kappa^{2} p^{2} m^{2}}{\mathrm{~N}^{2} e^{4}}, . . \cdot \text {. }
$$

so long at least as $\lambda$ is not less than about $4 \mu$.

* 'The Corpuscular Theory of Matter,' p. 84.

$\dagger$ Phil, Mag. vii. p. 165 (1904). 
This equation neglects resonance, and that this neglect is not justifiable is clear from the circumstance that in many of Rubens and Hagen's experiments the value of $C_{\lambda} / C_{\lambda}^{\prime}$ is less than unity. We shall avoid this difficulty, in so far as it can be avoided, by a method due to Schuster *. If wo allow for resonance, equation (15) must be replaced by

$$
\left(\frac{\mathrm{C}_{\lambda}}{\mathrm{C}_{\lambda}^{\prime}}\right)=1+\frac{\kappa^{2} p^{2} m^{2}}{\mathrm{~N}^{2} e^{4}}-\mathrm{S}
$$

where $\mathrm{S}$ is a term required by resonance, which, from the principle of conservation of energy, can be shown to be necessarily positive. It follows that the true value of $\mathrm{N}$ is necessarily less than the value calculated from equation (15).

12. No metal seems to be sufficiently regular in its optical behaviour in the infra-red to justify us in neglecting $S$ altogether. One of the most regular is platinum. For this Hagen and Rubens find $\dagger$

$$
\left(\frac{\mathrm{C}_{\lambda}}{\mathrm{C}_{\lambda}^{\prime}}\right)^{2}=20,1 \cdot 17 \text { at } \lambda=4 \mu, 8 \mu \text { respectively. }
$$

The corresponding upper limits for $\mathrm{N}$ are $2.3 \times 10^{23}$ and $2.7 \times 10^{23}$ respectively. To raise the temperature of $2 \cdot 3 \times 10^{23}$ electrons by $1^{\text {o }} \mathrm{C}$. requires $\cdot 75$ calorie, while to raise the temperature of 1 c.c. of platinum by $1^{\circ} \mathrm{C}$. requires only $\cdot 69$ calorie. Thus the greatest value for $\mathrm{N}$ permitted by the known specific heat of the substance is $2.1 \times 10^{23}$.

For gold, which is less regular in its optical behaviour, the upper limit given for $\mathrm{N}$ by equation (13) is $6 \times 10^{23}$, while the greatest value consistent with the known specific heat of gold is $1.9 \times 10^{23}$.

The substance for which our equations give the smallest upper limit for $\mathrm{N}$ is steel. From the reflecting power at $\lambda=8 \mu$, the upper limit for $N$ is $96 \times 10^{28}$, while the specific heat of steel admits a value as great as $3 \times 10^{23}$. But our theory has taken no account of the magnetic properties of the metals concerned, so that probably the upper limit obtained for $N$ on the supposition that $\mu=1$ for steel is untrustworthy.

Except for steel, there seems to be no substance for which the upper limit for $\mathbf{N}$ is not greater than the upper limit already set by the specific heat of the substance. In so far as our calculations assign a value for $N$ at all, these values are in general agreement with the law derived by Schuster $\ddagger$

* Phil. Mag. vii. p. 154 (1904)。

† I have used the numerical values given in Table II. p. 165, of the paper of Hagen and Rubens.

† Phil. Mag. vii. p. 151. (1904). 
from the application of Drude's equations to visible light: "the number of free electrons in a metal is equal to the number of atoms, or exceeds that number not more than three times." Or, if $n$ is the number of atoms per unit volume, $\mathrm{N}=p n$, where $p$ lies between 1 and 3. Assuming as we do that the electrons have energy appropriate to the temperature of the body, the law of Dulong and Petit sets the upper limit $2 \cdot 2$ to the value of $p$ for all metals, and a still smaller upper limit for those metals for which the atomic heat is less than 6.5 (e.g. for platinum, atomic heat $=6.29$, corresponding limit for $p$ assigned by specific heat is $2 \cdot 1$, our limit for $p$ is $2 \cdot 3$, Schuster's limit for $p$ is $1 \cdot 91$ ). When the limiting value for $p$ is found to be close to the limit allowed in this way by the specific heat, the inference is that almost all the heatenergy of the substance may reside in its free electrons. In such a case (if any such exists) the atoms must form an almost stationary network of obstacles through which the electrons move. We shall return to this later $(\S 23)$.

\section{Kirchhoff's Law.}

13. Let medium 1 be air and medium 2 be metal. Let a beam of radiation of frequency $p$ be incident on the boundary $A B$ of the media, bounded by cones of angles $\theta_{1}$ and $\theta_{1}+d \theta_{1}$ in medium 1 , and passing into medium 2 between cones $\theta_{2}$ and $\theta_{2}+d \theta_{2}$.

Fig. 1.

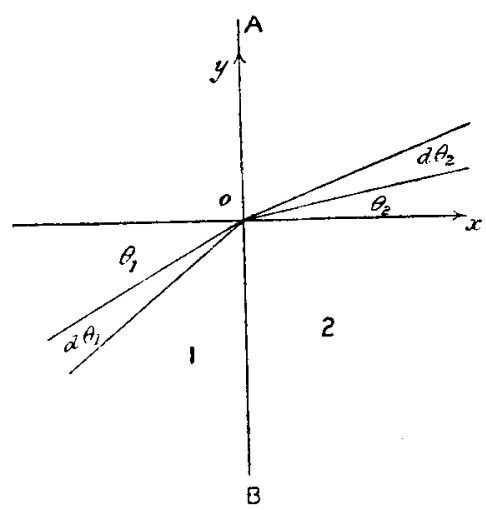

If $\mathrm{K}_{1}, \mathrm{~K}_{2}$ are the inductive capacities of the media, and $\mathrm{C}$ the velocity of radiation in vacuo, we have the equations

$$
\frac{\partial \gamma}{\partial y}-\frac{\partial \beta}{\partial z}=\frac{\mathrm{K}_{1}}{\mathrm{C}} \frac{d \mathrm{X}}{d t}, \& \mathrm{c}
$$


in air : and in the metallic medium

$$
\begin{aligned}
\frac{\partial \underline{\gamma}}{\partial y}-\frac{\partial \beta}{\partial z} & =\frac{\mathrm{K}_{2}}{\mathrm{U}} \frac{d \mathrm{X}}{d t}+4 \pi i_{x} \\
& =\frac{\mathrm{K}_{2}^{\prime}}{\mathrm{C}} \frac{d \mathrm{X}}{d t}, \text { \&c. . . . . }
\end{aligned}
$$

where $K_{2}^{\prime}$ is given by equation (14).

The velocity $V_{1}$ of radiation in medium 1 is given by $\mathrm{V}_{1}{ }^{2}=\mathrm{C}^{2} / \mathrm{K}_{1} \mu_{1}$. We introduce an angle $\theta_{2}{ }^{\prime}$ and a velocity $\mathrm{V}_{2}^{\prime}$ given by

$$
\frac{\sin ^{2} \theta_{2}{ }^{\prime}}{\sin ^{2} \theta_{1}}=\frac{\frac{1}{K_{2}^{\prime} \mu_{2}}}{\frac{1}{\mathrm{~K}_{1} \mu_{1}}}=\frac{\mathrm{V}_{2}{ }^{2}}{\mathrm{~V}_{1}{ }^{2}} . . . . .
$$

Clearly both $\sin \theta_{2}^{\prime}$ and $V_{2}^{\prime}$ will be complex, but their ratio is real.

The exponential through which the time and spacecoordinates enter in the radiation in medium (1) may be taken to be

$$
\text { exp. } i p\left(t-\frac{1}{\mathrm{~V}_{1}}\left(x \cos \theta_{1}+y \sin \theta_{1}\right)\right) \text {; }
$$

the corresponding factor in medium (2) will be

$$
\exp . i p\left(t-x \frac{\cos \theta_{2}{ }^{\prime}}{\mathrm{V}_{2}{ }^{\prime}}-y \frac{\sin \theta_{2}{ }^{\prime}}{\mathrm{V}_{2}^{\prime}}\right) \text {. . . }
$$

Remembering that $\frac{\sin \theta_{2}^{\prime}}{\bar{V}_{2}^{\prime}}$ is real, we see that it will be possible to introduce two new quantities $\theta_{2}$ and $V_{2}$, both real, such that

$$
\begin{aligned}
& \frac{\sin \theta_{2}{ }^{\prime}}{\mathrm{V}_{2}^{\prime}}=\frac{\sin \theta_{2}}{\mathrm{~V}_{2}} ; \\
& \frac{\cos \theta_{2}^{\prime}}{\bar{V}_{2}^{\prime}}=\frac{\cos \theta_{2}}{\mathrm{~V}_{2}}-i \epsilon .
\end{aligned}
$$

The exponential (18) now becomes

$$
e^{-\epsilon p x} \exp . i p\left(t-\frac{1}{\mathrm{~V}_{2}}\left(x \cos \theta_{2}+y \sin \theta_{2}\right)\right),
$$

and we now see that $\theta_{2}$ and $V_{2}$ are the angle of refraction and velocity of the radiation in medium 2 .

14. We introduce a new complex quantity $u_{12}$, associated with radiation passing from medium 1 to medium 2 , and 
given for vibrations polarized in the plane of incidence by

$$
u_{12}^{2}=\frac{\mathrm{K}_{2}^{\prime}}{\mu_{2}} \frac{\mu_{1}}{\overline{\mathrm{K}}_{1}} \frac{\cos ^{2} \theta_{\mathrm{a}}^{\prime}}{\cos ^{2} \bar{\theta}_{1}}, \quad \text {. . . . . }
$$

and for vibrations polarized perpendicular to the plane of incidence, by

$$
u_{12}^{2}=\frac{\mu^{2}}{K_{2}^{\prime}} \frac{K_{1}}{\mu_{1}} \frac{\cos ^{2} \theta_{2}^{\prime}}{\cos ^{2} \bar{\theta}_{1}^{\prime}} \cdot \text {. . . . . }
$$

Then if $R_{12}$ denotes the coefficient of reflexion of radiation incident at angle $\theta_{1}$ in medium (1), it can be shown in the usual way * that

$$
R_{12}=\left|\frac{1-u_{12}}{1+u_{12}}\right|^{2}
$$

where $|\alpha+i \beta|$ denotes the modulus of $\alpha+i \beta$, namely $\sqrt{\alpha^{2}+\beta^{2}}$.

For the coefficient of reflexion of radiation incident at angle $\theta_{2}$ in medium (2), we have similarly

$$
\mathrm{R}_{21}=\left|\frac{1-u_{21}}{1+u_{21}}\right|^{2} \text {, }
$$

where $u_{21}$ is given by equations similar to (19) and (20), but having $\mathrm{K}_{1}, \mu_{1}, \theta_{1}$ interchanged with $\mathrm{K}_{2}^{\prime}, \mu_{2}, \theta_{2}^{\prime}$. It is at once obvious that $u_{21}=\frac{1}{u_{12}}$, and from this it follows that $R_{12}=R_{21}$. It should be noticed that in this equation $R_{12}$ is evaluated for light incident at an angle $\theta_{1}$, but $R_{21}$ for light incident at an angle $\theta_{2}$.

15. Let media 1 and 2 be filled with radiation such that the energy per unit volume of radiation of frequency between $p$ and $p+d p$ is $\mathrm{E}_{1} d p$ in medium 1 and $\mathrm{E}_{2} d p$ in medium 2 .

The stream of energy which falls onto the boundary at an angle between $\theta_{1}$ and $\theta_{1}+d \theta_{1}$ per unit time is

$$
\frac{1}{2} \mathrm{E}_{1} \mathrm{~V}_{1} \cos \theta_{1} \sin \theta_{1} d \theta_{1} d p,
$$

so that the amount transmitted from medium 1 to medium 2 is

$$
\frac{1}{2} \mathrm{E}_{1} \mathrm{~V}_{1} d p \int\left(1-\mathrm{R}_{12}\right) \cos \theta_{1} \sin \theta_{1} d \theta_{1} . . .
$$

Similarly the amount transmitted from 2 to 1 is

$$
\frac{1}{2} \mathrm{E}_{2} \mathrm{~V}_{2} d p \int\left(1-\mathrm{R}_{21}\right) \cos \theta_{2} \sin \theta_{2} d \theta_{2}
$$

In a state in which there is an equal exchange of energy

* Jeans, ‘ Electricity and Magnetism,' p. 523. 
between the two media these expressions must be equal. Now we have, from equation (17),

$$
\frac{\sin \theta_{1}}{\mathrm{~V}_{1}}=\frac{\sin \theta_{2}^{\prime}}{\mathrm{V}_{2}^{\prime}}=\frac{\sin \theta_{2}}{\mathrm{~V}_{2}}
$$

and hence

$$
\frac{\cos \theta_{1} \sin \theta_{1} d \theta_{1}}{V_{1}^{2}}=\frac{\cos \theta_{2} \sin \theta_{2} d \theta_{2}}{\mathrm{~V}_{2}^{2}} .
$$

Since $R_{12}$ is the same function of $\theta_{1}$ as is $R_{21}$ of $\theta_{2}$, it follows that

$$
\frac{1}{\bar{V}_{1}^{2}} \int\left(1-R_{12}\right) \cos \theta_{1} \sin \theta_{1} d \theta_{1}=\frac{1}{V_{2}^{2}} \int\left(1-R_{21}\right) \cos \theta_{2} \sin \theta_{2} d \theta_{2},
$$

so that expressions (21) and (22) will be equal if

$$
\mathrm{E}_{1} \mathrm{~V}_{1}^{3}=\mathrm{E}_{2} \mathrm{~V}_{2}^{3} \text {. }
$$

This is a known result of which we have now obtained a proof by electrodynamical, without thermodynamical, principles.

16. The energy in a cavity in a body of any kind being of the form

$$
\mathrm{E}_{1}=f(\mathrm{~T}, p), . \quad . \quad . \quad . \quad .
$$

it follows that the energy in the interior of the body itself must be of the form

$$
\mathrm{E}_{2}=\left(\frac{\mathrm{V}_{1}}{\mathrm{~V}_{2}}\right)^{3} f(\mathrm{~T}, p), \ldots . . .
$$

so that the partition of energy inside matter can be determined in terms of black-body radiation. Using this value for $E_{2}$, the total emission per unit area per unit time from the surface of the body, by equation (22),

$$
\begin{aligned}
& =d p f(\mathrm{~T}, p) \frac{\mathrm{V}_{1}^{\prime}}{2 \mathrm{~V}_{2}^{2}} \int\left(1-\mathrm{R}_{21}\right) \cos \theta_{2} \sin \theta_{2} d \theta_{2} \\
& =d p f(\mathrm{~T}, p) \frac{1}{2} \mathrm{~V}_{1} \int^{2}\left(1-\mathrm{R}_{12}\right) \cos \theta_{1} \sin \theta_{1} d \theta_{1},
\end{aligned}
$$

by equation (23). Since $\theta_{1}$ is the angle the issuing radiation makes with the normal, the limits for $\theta_{1}$ are from 0 to $\overline{2}$. The coefficient of absorption A for light incident on the 
Motion of Electrons in Solids.

body is given by

$$
\begin{aligned}
\mathrm{A} & =\frac{\int_{0}^{\frac{\pi}{2}}\left(1-\mathrm{R}_{12}\right) \cos \theta_{1} \sin \theta_{1} d \theta_{1}}{\int_{0}^{\frac{\pi}{2}} \cos \theta_{1} \sin \theta_{1} d \theta_{1}} \\
& =2 \int_{0}^{\frac{\pi}{2}}\left(1-\mathrm{R}_{12}\right) \cos \theta_{1} \sin \theta_{1} d \theta_{1},
\end{aligned}
$$

so that the stream of issuing radiation is

$$
\frac{1}{4} \mathrm{~A} f(\mathrm{~T}, p) d p \text {. }
$$

Putting $A=1$ we pass to the case of an ideal perfectly black body, and find that the stream of issuing radiation must be $\frac{1}{4} f(\mathrm{~T}, p) d p$.

Hence, in general the stream issuing from any body is A times the stream issuing from a black body, as required by Kirchhoff's law.

We have derived Kirchhoff's law as a consequence purely of electron theory. It appears that the law is true quite independently of whether the rther is in equilibrium with matter or not: in fact the law is seen to be entirely independent of thermodynamic conditions of all kinds. It is consequently illegitimate to draw any thermodynamical inferences from the fact that Kirchhoff's law is observed to be true in nature.

\section{Emission of Radiation.}

17. If charges $e, e^{\prime}, \ldots$ move with velocities $\mathbf{u}, \mathbf{u},{ }^{\prime} \ldots$ there is no radiation if $\Sigma e \frac{d \mathbf{u}}{d t}=0$. If this condition is not satisfied, let $\mathrm{EU}$ be the vector which is the resultant of the vectors $e \mathbf{u}, e^{\prime} \mathbf{u}^{\prime}, \ldots$ Then

$$
-\mathrm{E} \frac{d \mathbf{U}}{d t}+\Sigma e \frac{d \mathbf{u}}{d t}=0
$$

so that the radiation from $\mathrm{E}, e, e,{ }^{\prime} \ldots$ is nil. Hence the radiation from $e, e,{ }^{\prime} \ldots$ moving with velocities $\mathbf{u}, \mathbf{u},{ }^{\prime} \ldots$ is equal to and identical with that of a single charge $E$ moving with velocity U. In this result it has to be supposed that the distances apart of the charges $e, e,{ }^{\prime} \ldots$ are small compared with the wave-length of the emitted radiation, or at least of that part of it with which we are concerned. 
Let us take an element of volume $d v$ which contains a great number of electrons $\mathrm{N} d v$, and let us suppose for the present that the linear dimensions of $d v$ are small compared with the wave-length of the light with which we are concerned. Let these electrons have velocities of components

$$
u, v, w ; u^{\prime}, v^{\prime}, w^{\prime} \ldots,
$$

and let the mean of these components, as before, be $u_{0}, v_{0}, w_{0}$.

Then we can replace the radiation from these electrons by the radiation from an electron of charge $\mathrm{N} e d v$ having velocity components $u_{0}, v_{0}, w_{0^{*}}$. If $f$ is the acceleration of this electron, the radiation from it in time $t$ is *

$$
\int_{0}^{t} \frac{2 \mu}{3 \mathrm{~V}}(\mathrm{~N} e d v f)^{2} d t
$$

The components of the total current $i$ in the element $d v$ are

$$
\mathrm{N} e d v u_{0}, \mathrm{~N} e d v v_{0}, \mathrm{~N} e d v w_{0}
$$

so that we have

$$
\mathrm{N} e d v f=\frac{d i}{d t}
$$

and the radiation in time $t$ is

$$
\frac{2 \mu}{3 \mathrm{~V}} \int_{0}^{t}\left(\frac{d i}{d t}\right)^{2} d t
$$

We can express $\frac{d i}{d t}$ in a Fourier-series in the form

where

$$
\frac{d i}{d t}=\frac{1}{\pi} \int_{p=0}^{p=\infty}\left(\mathrm{A} p \cos p t+\mathrm{B}_{p} \sin p t\right) d p
$$

$$
\left.\begin{array}{l}
\mathrm{A}_{p}=\int_{0}^{t} \frac{d i}{d t} \cos p t d t \\
\mathrm{~B}_{p}=\int_{0}^{t} \frac{d i}{d t} \sin p t d t,
\end{array}\right\} . . . .
$$

* Here $\mathrm{V}$ is the velocity in the medium, and $\mu$ is the magnetic permeability. The result is easily obtained by mudifying Larmor's calculation ('Ather and Matter,' p. 227).

The radiation is calculated by integrating over a sphere of large radius, so that we must imagine all absorbing and dispersing electrons remuved from inside this sphere. Thus V is not the velocity in the actual medium, but in the medium when freed from absorption and dispersion. It is given by $\mathrm{V}^{2}=\mathrm{C}^{2} / \mathrm{K} \mu$, and becomes identical with $\mathrm{V}_{2}$, the actual velocity of waves in the medium, when $p=0$. 
and now have *

$$
\int_{0}^{t}\left(\frac{d i}{d t}\right)^{2} d t=\frac{1}{\pi} \int_{0}^{\infty}\left(\mathrm{A}_{p}^{2}+\mathrm{B}_{p}^{2}\right) d p .
$$

The total emission of the element $d v$ in time $t$ is accordingly

$$
\frac{2 \mu}{3 \pi \mathrm{V}} \int_{0}^{\infty}\left(\mathrm{A}_{p}^{2}+\mathrm{B}_{p}^{2}\right) d p, \ldots . .
$$

and in this expression the coefficient of $d p$ represents the emission of radiation of frequency between $p$ and $d p$.

Instead of (27) we may take

$$
\begin{aligned}
\mathbf{A}_{p} & =\int_{0}^{t} \frac{d i}{d t} \cos p t d t \\
& =|i \cos p t|_{0}^{t}+p \int_{0}^{t} i \sin p t d t ;
\end{aligned}
$$

when $t$ is large enough, the first term may be ignored, and we may take

$$
\begin{aligned}
& \mathrm{A}_{p}=p \int_{0}^{t} i \sin p t d t, . . \\
& \mathrm{B}_{p}=-p \int_{0}^{t} i \cos p t d t . \quad . \quad . \quad .
\end{aligned}
$$

The calculation of the emission requires the evaluation of these integrals.

18. Let us first perform the calculation on the simplifying assumption that the time $t$ can be divided into $n$ equal treepath periods each of time $\tau$, and let us suppose that at the end of each of these periods the velocities of the electrons are replaced by new velocities which have no reference to the old. We have, by equation (29),

$$
\begin{aligned}
\mathrm{A}_{p} & =p \sum_{s=0}^{s=n-1} \int_{0}^{\tau} i \sin p(t+s \tau) d t \\
& =\sum_{s=0}^{s=n-1} 2 i \sin p\left\{t+\left(s+\frac{1}{2}\right) \tau\right\} \sin \frac{1}{2} p \tau,
\end{aligned}
$$

so that, since there is no relation between the values of $i$ on

* Rayleigh, Phil. Mag. [5] xxvii. p. 466. 
the different free-path periods,

and consequently

$$
\begin{aligned}
& \mathrm{A}_{p}^{2}=\sum_{s=0}^{s=n-1} 4 i^{2} \sin ^{2} p\left\{t+\left(s+\frac{1}{2}\right) \tau\right\} \sin ^{2} \frac{1}{2} p \tau \\
& \mathrm{B}_{p}^{2}=\sum_{s=0}^{s=n-1} 4 i^{2} \cos ^{2} p\left\{t+\left(s+\frac{1}{2}\right) \tau\right\} \sin ^{2} \frac{1}{2} p \tau,
\end{aligned}
$$

$$
\mathrm{A}_{p}^{2}+\mathrm{B}_{p}^{2}=4 n i^{2} \sin ^{2} \frac{1}{2} p \tau, \quad . \quad . \quad . \quad .
$$

where $i^{2}$ now denotes the average value of $i^{2}$ in the different intervals.

We can easily evaluate $i^{2}$. The $x$-component of $i$ is given by

so that

$$
i_{x}=e\left(u+u^{\prime}+\ldots\right) \text {, }
$$

$$
i_{x}^{2}=e^{2}\left(u^{2}+u^{\prime 2}+\ldots\right)=\mathrm{N} d v e^{2} \frac{\mathrm{RT}}{m}
$$

and hence

$$
i^{2}=3 \mathrm{RT} \frac{\mathrm{N} e^{2}}{m} d v . \quad . \quad . \quad . \quad . \quad .
$$

Substituting in expression (28) from equations (31) and (32), the emission in time $t$ is found to be

$$
\left(\int \frac{8 \mu}{\pi \mathrm{V}} \frac{\sin ^{2} \frac{1}{2} p \tau}{\tau} \frac{\mathrm{N} e^{2}}{m} \mathrm{RT} d p\right) t d v \ldots .
$$

This expression is proportional to $t$ and to $d v$ as it ought to be. We notice also that it vanishes if $\tau$ is either very great or very small, so that when the motion is regarded as made up of free-paths, the whole phenomenon of emission depends on the free-path being finite.

From the formula for the emission we can readily calculate the partition of radiant energy in the matter in the steady state in which emission and absorption are equal to one another. Let the energy in this state be

$$
\int \mathrm{E}_{p} d p
$$

per unit volume. Then, as Thomson shows*, the absorption of energy in the element $d v$ in time $t$ must be

$$
\left(\int \frac{4 \pi \mathrm{C}^{2}}{\mathrm{~K}} c \mathrm{E}_{p} d p\right) t d v, \quad . \quad . \quad .
$$

where $c$ is the conductivity of the medium for currents of

* Phil. Mag. xiv. p. 223. 
frequency $p$. The proper value to use for $c$ when we suppose the motion made up of free-paths of equal duration is that given by Thomson, namely (in our notation)

$$
c=2 \frac{\sin ^{2} \frac{1}{2} p \tau}{p^{2} \tau} \frac{\mathrm{N} e^{2}}{m} .
$$

Using this value of $c$, and equating the emission (33) to the absorption (34), we obtain

$$
\mathrm{E}_{p}=\frac{p^{2} \operatorname{RTK} \mu}{\pi^{2} \mathrm{C}^{2} \mathrm{~V}}=\frac{p^{2} \mathrm{RT}}{\pi^{2} \mathrm{~V}^{3}}, . . \quad .
$$

19. We next examine how this must be modified when we do not assume the existence of free-paths.

If $t_{1}, t_{2}$ are any instants within the interval from 0 to $t$, and if $i_{1}, i_{2}$ are the values of $i$ at these instants, we have, by equation (29)

$$
\mathrm{A}_{p}=p \int_{0}^{t} i_{1} \sin \rho t_{1} d t_{1}=p \int_{0}^{t} i_{2} \sin p t_{2} d t_{2}
$$

so that, by multiplication,

$$
\mathrm{A}_{p}^{2}=p^{2} \int_{0}^{t} \int_{0}^{t} i_{1} i_{2} \sin p t_{1} \sin p t_{2} d t_{1} d t_{2}
$$

Similarly from equation (30)

and by addition

$$
\mathrm{B}_{p}^{2}=p^{2} \int_{0}^{t} \int_{0}^{t} i_{1} i_{2} \cos p t_{1} \cos p t_{2} d t_{1} d t_{2}
$$

$$
\begin{aligned}
\mathrm{A}_{p}^{2}+\mathrm{B}_{p}^{2} & =p^{2} \int_{0}^{t} \int_{0}^{t} i_{1} i_{2} \cos p\left(t_{1}-t_{2}\right) d t_{1} d t_{2} \\
& =p^{2} \int_{t_{1}=0}^{t_{1}=t} \int_{t_{2}=0}^{t_{2}=t} i_{1} i_{2} \cos p\left(t_{1}-t_{2}\right) d\left(t_{1}-t_{2}\right) d\left(\frac{t_{1}+t_{2}}{2}\right) \cdot(36)
\end{aligned}
$$

Let the two instants $t_{1}, t_{2}$ be at equal intervals $\theta$ from their middle instant $t^{\prime}$ of which the value is $\left(\frac{t_{1}+t_{2}}{2}\right)$. Let $i^{\prime}$ be the current at $t^{\prime}$. The expectation of rate of change of $i$ is (cf. equation (8))

$$
\frac{d i}{d t}=-\frac{\mathrm{N} e^{2}}{m \kappa} i, . . . . . .
$$

of which the integral is

$$
i=i_{0} e^{-\epsilon t}, \quad \cdot \quad \cdot \quad \cdot \quad \text {. }
$$

Phil. Mag. S. 6. Vol. 17. No. 102. June 1909. $3 \mathrm{H}$ 
where $\epsilon=\frac{N e^{2}}{m \kappa}$. Hence, knowing the current $i$ at time $t^{\prime}$, the value of $i_{2}$, the current at time $t_{2}$, i.e. after an interval $\theta$, is

$$
i_{2}=i^{\prime} e^{-\epsilon \theta}+j \text {, }
$$

where $j$ is a quantity of which the "expectation" is zero. Similarly, since the motion is reversible, the value of $i_{1}$ is

$$
i_{1}=i^{\prime} e^{-\epsilon \theta}+j^{\prime} \text {, }
$$

where $j^{\prime}$ has expectation zero, and has no correllation with $j$. Putting in these values, equation (36) becomes

$$
\mathrm{A}^{2}+\mathrm{B}^{2}=p^{2} \int i^{\prime 2} e^{-2 \theta \theta} \cos 2 p \theta d(2 \theta) d t^{\prime}, . .
$$

the terms in $j, j^{\prime}$ and $j j^{\prime}$ being omitted because, as their average value is zero, they vanish on integration. The exponential $e^{-2 \epsilon \theta}$ vanishes very rapidly as the interval $2 \theta$ between $t_{1}$ and $t_{2}$ increases-this is the mathematical expression of the fact that there is very little correlation between the values of $i$ at intervals of time far apart. Hence we may integrate with respect to $\theta$ from $-\infty$ to $+\infty$, taking $\theta$ always positive in the exponential $e^{-2 \epsilon \theta}$, and so obtain

$$
\begin{aligned}
\mathrm{A}^{2}+\mathrm{B}^{2} & =p^{2} \int_{t^{\prime}=0}^{t^{\prime}=t} i^{\prime 2} \frac{2 \epsilon}{\epsilon^{2}+p^{2}} d t^{\prime} \\
& =\frac{2 p^{2} \epsilon}{\epsilon^{2}+p^{2}} 3 \mathrm{RT} \frac{\mathrm{N} e^{2}}{m} t d v,
\end{aligned}
$$

by equation (32). Replacing $\epsilon$ by its value, this gives the emission of the element $d v$ in the form ( $f$. equation (28j)

$$
\left(\int \frac{4 \mu}{\pi \mathrm{V}} \frac{\kappa}{1+\frac{\kappa^{2} p^{2} m^{2}}{\mathrm{~N}^{2} e^{4}}} p^{2} \mathrm{RT} d p\right) t d v .
$$

This, as before, must be equal to the absorption given by expression (34). The proper value for $c$ is now that calculated for continuous motion, and given in equation (10). Thus the absorption must be

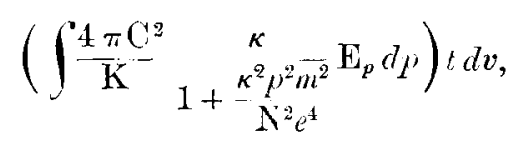


and equating this to expression (41) we obtain

$$
\mathrm{E}_{p}=\frac{p^{2} \mathrm{RTK} \mu}{\pi^{2} \mathrm{C}^{22} \mathrm{~V}}=\frac{p^{2} \mathrm{RT}}{\pi^{2} \mathrm{~V}^{\overline{3}}}, . . .
$$

which is exactly the value obtained before (equation (35)).

20 . We notice that $\mathrm{E}_{p}$ depends on the structure of the me dium only through the factor $1 / \mathrm{V}^{3}$. This is as it should be, by equation (26), to accord with the experimental fact tha the radiation in a cavity is independent of the nature of the matter. From equations (25), (26), and (41), it follows that the radiation in the cavity must be given by

$$
\mathrm{E}_{p}=\frac{p^{2} \mathrm{RT}}{\pi^{2} \bar{V}_{1}^{3}}, \quad . \quad . \quad . \quad . \quad .
$$

where $V_{1}$ is the velocity in the cavity.

If $\lambda$ is the wave-length of radiation of frequency $p$ we have $p \lambda=2 \pi V$ in metal, and $=2 \pi V_{1}$ in the cavity. The radiation $\int \mathrm{E}_{p} d p$ in the metal, $\mathrm{E}_{p}$ being given by equation (41), accordingly transforms into

$$
\int 8 \pi \mathrm{RT} \lambda^{-4} d \lambda, \quad \text {. . . . . }
$$

when expressed in terms of $\lambda$, and the energy (42) in air transforms into exactly the same expression. Moreover, expression (43) expresses the partition of energy demanded by the law of equipartition *, both for the metal and the cavity.

21. When dealing with waves of shorter wave-length the principal modification which has to be made originates in the need for changing equation (38). Other modifications are needed which alter the radiation by an appreciable fraction of its whole amount, but it is easily seen that this particular modification changes the order of magnitude of the radiationt.

For, by $\$ 5$, when $p$ is very great, we must replace equation (38), namely

$$
i=i_{0} e^{-\epsilon t},
$$

by an equation of the form ( $c f$. equation $(5))$

in which $\frac{\partial \phi}{\partial t}=0$ when $t=0$.

$$
i=i_{0} \phi(t) \text {, }
$$

* Phil. Mag. xvii. p. 231.

t These remarks apply only to natural radiation, and not to the radiation inside an ideal perfectly-reflecting enclosure. For this latter radiation it will be proved, in the second part of this papor, that equations (38) and (43) are true throughout the whole spectrum. 
Thus in equation (39), instead of an integral of the form

$$
\int e^{ \pm 2 \varepsilon \theta} \cos 2 p \theta d(2 \theta), \text {. . . . . }
$$

we have an integral of the form

$$
\begin{gathered}
\int \phi(2 \theta) \cos 2 p \theta d \\
\text { integral }(44) \text { is } \\
\frac{2 \varepsilon}{\epsilon^{2}+p^{2}},
\end{gathered}
$$

but, in virtue of the fact that $\frac{d \phi}{d t}=0$ when $t=0$, the value of the integral (45) falls off as $e^{-\beta p}$ (where $\beta$ is independent of $p$ ) when $p$ is very great*.

This is perfectly in accordance with observation, and it seems to be as far as the theory can be carried without introducing special laws of force between electrons and matter. This will be done in another paper.

\section{Conclusion.}

22. The result of $\S 20$ seems to prove beyond reasonable doubt that there is equipartition of energy between the different vibrations of great wave-length, both in the interior of the matter and in a cavity in the matter.

Each vibration has the energy appropriate to a temperature $T$ which has been introduced into our analysis as the temperature determined by the kinetic energy of the free electrons.

Two pieces of evidence identify this temperature $\mathrm{T}$ with what we call the temperature of the matter. There is first the evidence provided by the observed energy of radiation of great wave-length + ; there is, secondly, the evidence provided by the observed energy of the electrons escaping from hot metals $\ddagger$.

Thus we can say that the temperature of a solid is defined equally well either by

(i.) the mean energy of vibrations of great wave-length in its interior ; or

(ii.) the mean kinetic energy of the free electrons in its interior.

* 'Dynamical Theory of Gases,' $\$ \$ 237-240$.

† Lummer and Pringsheim, Verhand. d. deutscher phys. Gessellschaft, 1900 , p. 163.

¥ Richardson and Brown, Phil. Mag. xvi. p. 353. 
We have seen that these two definitions lead to the same temperature. The first definition explains at once the tendency for the temperatures of two bodies to equalise by heatradiation; the second definition explains the tendency for their temperatures to equalise by conduction.

In a gas the temperature is defined in only one way, namely, by the mean energy of translation of its molecules. The question arises as to whether the temperature of a solid can also be defined in a similar way.

So far as present evidence goes, it seems as though this question must be answered in the negative, for the following reasons.

23. For almost all metals the atomic heat is, to within a few per cent., equal to $5 \cdot 88$, the value required if each atom had associated with it energy $3 \mathrm{RT}-i . e$. twice the translational energy of a free electron or molecule of gas at temperature $\mathrm{T}$.

If the energy of motion of the atom is governed by the temperature $T$, each atom must, on account of this motion, have associated with it energy exactly equal to $3 \mathrm{RT}$, half of this being contributed by its average kinetic energy ( $\left.{ }_{2}^{2} \mathrm{RT}\right)$, and half by its average potential energy ( $R T$ ). But it is difficult to imagine the atoms moving freely as regards translational motion without at the same time being set into rotation, and the energy of this rotation, if governed by the temperature T, would be 3RT per atom. Each atom would now have energy $6 \mathrm{RT}$ associated with it*.

Further, each atom has associated with it a number of free electrons of which the average, according to Schuster's table $t$, is about two per atom. This adds a further contribution 3RT to the energy to be associated with each atom.

Thus the energy per atom would, under these circumstances, seem to be about $9 R T$, made up of three equal contributions of 3RT each from motion of atoms, rotation of atoms, and motion of electrons. The value permitted by the specific heats is uniformly 3RT. The uniformity of this number indicates. that we must attribute it to a similar origin in all substances.

It seems as if the only permissible view is that the $3 \mathrm{RT}$ is contributed by the motion of electrons. If we accept this, the rejection of the contribution $6 \mathrm{RT}$ from the atoms means that the energy of motion of these is very small in comparison

* If we take the molecule as unit, each molecule would hare energy 6RT, and therefore each atom, at least in diatomic substances, would have energy 3RT, the required amount. But Dulong and Petit's law is not limited to diatomic elements, and seems to show conclusively that the atom must be taken as unit.

+ Phil. Mag. vii. p. 155. 
with what it would be if governed by the temperature. The atoms now form a framework of obstacles through which the electrons move. This framework is continually set into vibration by collisions with the electrons, but its motion is dissipated into radiation so rapidly that the atoms never acquire a motion comparable with temperature-motion.

This view compels us to suppose that each atom has always two electrons associated with it*. The whole of these electrons need not be tree at all temperatures. In some substances which are poor conductors, only a few electrons may be free, and in raising the temperature a large part of the energy may be used in setting free new electrons. For such substances the atomic heat need not be near $5 \cdot 88$, but it should approximate to this valne as we reach temperatures at which all the electrons have become free. Weber's researches $\dagger$ show that such an asymptotic limit exists. Here, for instance, is the variation of the atomic heat of carbon (diamond):-

$\begin{array}{ccccccccc}\text { Temperature ... } & 0^{\circ} & 50^{\circ} & 100^{\circ} & 150^{\circ} & 200^{\circ} & 606^{\circ} & 806^{\circ} & 985^{\circ} \\ \text { Atomic heat ... } & 1.12 & 1.72 & 2 \cdot 28 & 281 & 333 & 5 \cdot 26 & 5.36 & 5.49\end{array}$

The energy of motion of the atoms, although small, need not be altogether negligible, so that the limit reached when all the electrons have been set free, may be some what greater than the exact value $5 \cdot 88$. As the melting-point is approached, the energy of the atoms will increase greatly, so that this value may be much exceeded. Thus Pionchon $\ddagger$ finds for the atomic heat of iron at $500^{\circ}$ the value $9 \cdot 84$.

24. This view of the matter is still not completely satisfactory, for it leaves it difficult to understand the process of conduction of heat between a solid and a gas in contact with it. It seems as if we must suppose the atoms to form into large clusters, which themselves take up the temperaturemotion from the electrons and might convey it to the molecules of a gas in contact. The motion of these clusters as they are bombarded by the electrons, would be similar to that of the solid particles in the Brownian movements. The energy of motion of the clusters, namely, 3RT or $6 \mathrm{RT}$ for each, would only contribute imperceptibly to the value of the specific heat.

If this last conjecture is correct there would be another way of defining the temperature of a solid in addition to the two already given; we might define it by the mean kinetic energy of its molecular clusters.

January $7,1909$.

* Except perhaps in elements whose atomic weight has been calculated solely from the specific heat.

+ Pogg. Ann. cliv. p. $575 . \quad+$ C. R. cvi. p. 1344. 